\title{
Research on the Combination of Non-binary LDPC Code with OFDM and OVXDM System
}

\author{
Zhang Fan ${ }^{1, a}$, Tu De-feng ${ }^{2, b}$ \\ 1. Science and Technology Research Bureau, Anhui Xinhua University, Hefei, China \\ 2. School of Electronic Communication Engineering, Anhui Xinhua University, Hefei, China \\ a.salas0527@163.com, b.286072809@qq.com
}

\begin{abstract}
Compared with the binary LDPC code, the multi-domain LDPC code can improve the error-correcting performance by choosing the non-zero element to increase the column weight of the equivalent binary LDPC code under the condition that the binary diagram structure of the binary LDPC code with the equivalent code length remains unchanged. When the channel is in deep fading, if channel coding and interweaving technology are applied to OFDM and OVXDM systems, these sudden errors can be corrected and the performance of the system can be further improved. On the basis of introducing the excellent characteristics of OFDM, OVCDM and OVTDM, the author tries to build a system model that combines the multi-domain LDPC code with these technologies, and tries to make an in-depth study on data transmission and coding.
\end{abstract}

Keywords: non-binary LDPC Code; OFDM; OVCDM; OVTDM

\section{Introduction}

Multiple LDPC code have advantages over binary LDPC code, which can ensure that the error correction performance can be improved by choosing non-zero element to increase the column weight of equivalent binary LDPC code under the condition that the binary graph structure of binary LDPC code with equivalent code length remains unchanged[1].

OFDM (Orthogonal Frequency Division Multiplexing) is actually a type of MCM (Multi Carrier Modulation). The parallel transmission of high speed serial data is realized by frequency division multiplexing, which has good resistance to multipath weakness and can support multi-user access. OVXDM (Overlapped X Division Multiplexing) is the overlapping Multiplexing system of X domain (X stands for any domain, time $\mathrm{T}$, code $\mathrm{C}$, space $\mathrm{S}$, frequency $\mathrm{F}$ or hybrid $\mathrm{H}$, etc.)[2]. Due to limited space, it is only discussed OVCDM and OVTDM in this paper.

When the channel is in deep fading, if channel coding and interleave technology are used in OFDM and OVXDM systems, it can help correct the burst errors of ICI and ISI caused by multipath fading, and further improve the system performance. In this paper, the project team tries to build a system model that combines multi-domain LDPC code with OFDM and OVXDM technology, and makes a beneficial exploration on data transmission and coding.

\section{Multiple LDPC Coding and Decoding System}

LDPC code is a packet error correcting code with sparse check matrix proposed by Robert Gallager of Massachusetts Institute of Technology in his doctoral dissertation in 1962. It is applicable to almost all channels, so it has become a research hotspot in coding field in recent years. Its performance is close to Shannon limit, and the description and implementation is simple. And it is easy to theoretical analysis and research, whose decoding is simple and can be implemented in parallel operation. So it is very suitable for hardware implementation. 


\section{A. Features of LDPC}

Any $(n, k)$ block code that can be described by a linear equation is called a linear block code if the relationship between the information element and the supervisor is linear. A low density parity code graph (LDPC code) is essentially a linear block code that maps a sequence of information to a sending sequence, or code word sequence, through a generation matrix $\mathrm{G}$. For the generation matrix $\mathrm{G}$, there exists exactly equivalent parity matrix $\mathrm{H}$, and all code word sequences $\mathrm{C}$ constitute the null space of matrix $\mathrm{H}$.

The parity check matrix H of LDPC code is a sparse matrix. Relative to the length of rows and columns, the number of non-zero elements in each row and column of the check matrix is very small, which is why LDPC code is called low-density code. Due to the sparsity of the check matrix $\mathrm{H}$ and the different rules used in the construction, the coded binary graph (Taner Graph) of different LDPC code has different closed loop distributions. The closed loop in the bipartite graph is an important factor affecting the performance of LDPC code, which makes LDPC code show completely different decoding performance under a kind of iterative decoding algorithm like the Belief Propagation algorithm.

\section{B. Coding and Decoding Characteristics of Multiple LDPC}

The definition of LDPC code is based on the binary domain. MaKcay popularized the LDPC code of the binary domain. LDPC code on GF(q) of multiple domains can be obtained if the fields defined are not limited to binary fields. LDPC code on multiple domains have better performance than binary LDPC code, and practice shows that LDPC code constructed on larger domains have better decoding performance. For example, the performance of regular code constructed on GF(16) is similar to that of Turbo code. The reason why multi-domain LDPC code has such excellent performance is that it has a heavier column weight than the binary domain LDPC code, and at the same time it has a bipartite graph structure similar to that of the binary domain LDPC code.

\section{OFDM System}

OFDM is a key technology that can effectively counter the inter-code interference caused by high-speed data transmission and channel multipath fading. OFDM transmits high-speed data through a series and modulates it over a number of mutually orthogonal subcarriers.

\section{A. Features}

The characteristics of Orthogonal frequency division multiplexing (OFDM) is a special kind of multi-carrier transmission technology, which can be effectively against multipath fading and overcome inter symbol interference and can realize high speed data transmission at high frequency spectrum utilization. So it can significantly reduce the computation complexity, which has been widely used in the digital audio broadcasting (DAB), digital video broadcasting (DVB), wireless LAN (WLAN), and other technical areas. However, a major defect of OFDM signal is that the peak average power is higher than PAPR, which limits the wide application of OFDM system. When the input data at the input end are in the same phase, the output signal of OFDM system will have a large peak value. Although the probability of high PAPR is very low, the high power amplifier (HPA) and $\mathrm{A} / \mathrm{D}$ converter of the system are required to transmit these OFDM signals without distortion. If nonlinearity occurs at peak power, intermodulation interference and out-of-band radiation between subcarriers will occur, which will greatly reduce the system performance of OFDM. Therefore, it is important for OFDM systems to adopt appropriate techniques to reduce PAPR.

\section{B. Common OFDM Technology}

\section{1) $C O F D M$}

OFDM can solve channel selective fading well in multipath environment, but it has not been well overcome channel flat fading (that is, the amplitude of each carrier obeys Rayleigh distribution).The OFDM used channel coding to solve this problem is called COFDM (Coded OFDM).The basic 
principle is to combine frequency-selective fading channel (frequency domain) with time-varying flat fading channel (time domain) to form time-frequency domain.

2) $M I M O-O F D M$

MIMO (multiple-input multiple-output) technology, first proposed by Marconi in 1908, which used multiple antennas to suppress channel fading. MIMO systems can take advantage of multipath components in propagation to a certain extent, which means that MIMO can resist multipath fading. But for frequency-selective deep fading, MIMO systems are still powerless. At present, the solutions to frequency-selective fading in MIMO systems are usually equalization and OFDM. Most researchers believe that OFDM technology is the core technology of $4 \mathrm{G}$, and $4 \mathrm{G}$ requires the technology of very high spectrum utilization. After all, OFDM's role in improving spectrum utilization is limited. Rational development of space resources on the basis of OFDM, namely MIMO+OFDM, can provide higher data transmission rate.

\section{3) $M I M O-O F D M$}

BICM (Bit Interleaved Code Modulation) is a Bit Interleaved encoding. BICM is one of the key technologies in MIMO wireless communication, which can improve the time diversity effect of coding modulation and the error performance of communication system by connecting errorcorrecting coding, bit-level interleaver and higher-order modulation in serial. Tonello applies BICM and iterative decoding to space-time joint coding modulation (namely BICM-OFDM). These systems use convolutional code or Turbo code as the outer code, and the receiver adopts Turbo receiving criterion, namely demodulator (detector) and decoder for iterative reception.

\section{OVXDM System}

OVXDM (Overlapped X Division Multiplexing) is the overlapping Multiplexing system of X domain (X stands for any domain, time $T$, code $C$, space $S$, frequency $F$ or hybrid $H$, etc.) In this paper, only OVCDM and OVTDM are discussed.

\section{A. OVCDM}

Overlapping code division multiplexing system is a generalized convolutional coding system, and its system model can be expressed as follows:

$$
\mathrm{V}_{n}^{T}=\boldsymbol{F}\left(\sum_{l=0}^{\operatorname{Min}(n, L-1)} \mathbf{U}_{n-l}^{T} \mathbf{B}_{l}\right)
$$

Where $\mathrm{F}(*)$ represents the monotone nonlinear transformation with one-to-one correspondence to *. $\mathbf{U}_{n}^{T}=\left[\tilde{u}_{n, 0}, \tilde{u}_{n, 1}, \ldots, \tilde{u}_{n, k-1}\right]^{T}$ is K-dimensional parallel complex data vector. The encoding matrix of order $\mathrm{K}^{*} \mathrm{LN}$ is expressed as $\boldsymbol{B}=\left[\mathrm{B}_{0}, \mathrm{~B}_{1}, \ldots, \mathrm{B}_{L-1}\right]$. See Figure 1. for the working principle diagram of the corresponding system[3].

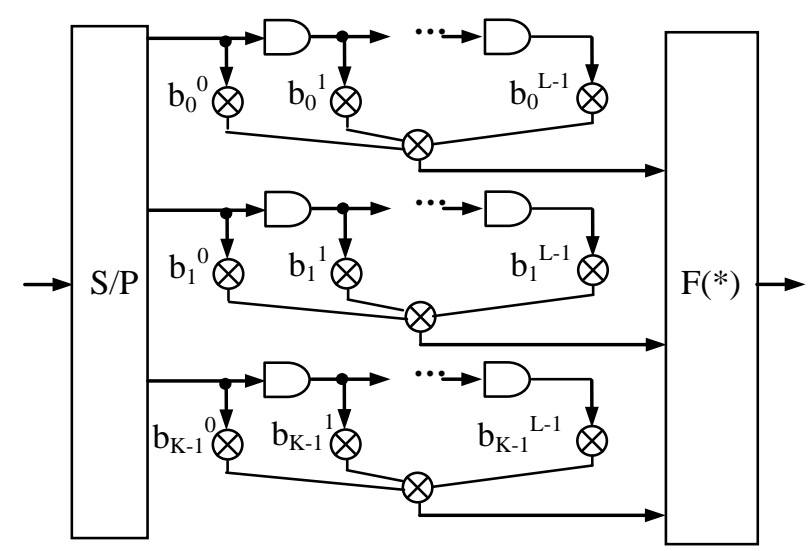

Figure 1. OVCDM system model

OVCDM is multiplexed through overlapping code in complex fields, and its coded output approximates the Gaussian distribution. According to Shannon's coding theorem, under the 
condition of Gaussian white noise channel, only the input signal satisfying the Gaussian distribution can reach the channel capacity, which precisely meets this condition. Moreover, there is always a one-to-one correspondence between the encoding input sequence and the encoding output. Therefore, the spectral efficiency of the system can be greatly improved by using the linear or nonlinear convolutional encoding multiplexing (multiple access) structure. At the same time, when the appropriate coding matrix is selected, the system has obvious coding gain compared with the traditional high-order modulation with the same spectral efficiency.

\section{B. OVTDM}

According to the information theory, in addition to the maximization of the distance between the output sequences, a good coding output should also present a complex Gaussian distribution when the channel noise presents a complex Gaussian distribution. However, most of the current encoding is carried out in the finite domain, and the output of the encoding must be modulated to realize the output of the complex domain. In addition, the constellation points realized by the current modulation are uniformly linked, and the converted complex domain signals are uniformly linked, which is far from the complex Gaussian distribution of the optimal encoded output. Although it is possible to concentrate the constellation points using the technique, the gain from this repair is limited.

When designing the coding scheme, we want the number of levels to be as small as possible. The number of levels required for existing coded modulation increases exponentially. The using of technology also merges some constellation points, but the reduction of points is very limited. Therefore, under the condition of limited average power, the more constellation points, the smaller Euclidean distance between points, which reduced anti-interference ability under the interference of additive noise.

OVTDM solves the above two problems well. In addition to the advantage of approximating the Gaussian distribution in output, the number of output levels is also reduced. Further construction of the serial cascade system, it has faster approximation of the complex Gaussian distribution, and reduce the required number of levels. In addition to very high spectral efficiency, and it has a good coding gain.

Set the symbol sequence of signal to be sent as:

$$
S=\left\{\mathrm{s}_{0}, \mathrm{~s}_{1}, \ldots, \mathrm{s}_{N-1}\right\}
$$

Then the equivalent baseband signal of OVTDM can be expressed as

$$
x(t)=\sum_{n=0}^{N-1} s_{n} g(t-n \Delta t)
$$

Where, $g(t)$ is the impulse response of the shaping filter. When $t<0$ or $t>t, g(t)=0$. If $x(t)$ is sampled at integer multiples of $\Delta t$, it is obtained

$$
x(m)=\sum_{n=0}^{N-1} s_{n} g(m-n)=\sum_{n=m-L+1}^{m} s_{n} g(m-n)=\sum_{l=0}^{L-1} s_{m-l} g(I)
$$

Where, $0 \leq \mathrm{m}<\mathrm{N}+\mathrm{L}-1$, it is the $\mathrm{L}$ sample values of $\mathrm{g}(\mathrm{t})$ on $[0, \mathrm{~T})$. Thus, the convolutional encoding model of OVTDM signal can be obtained, as shown in Figure 2.

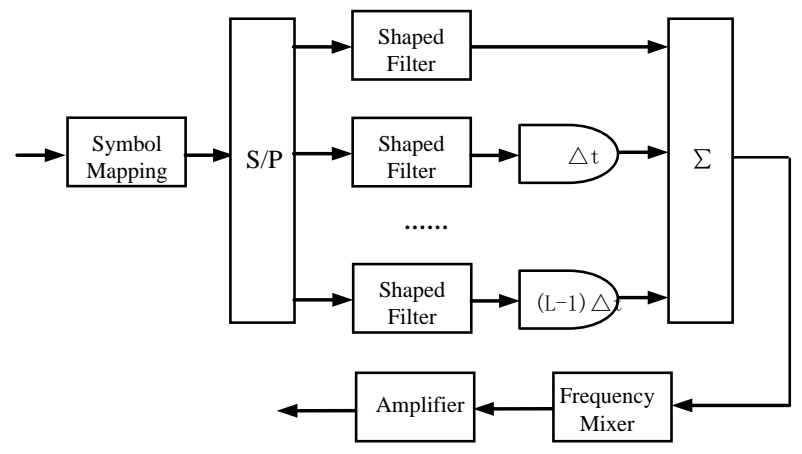

Figure 2. $\quad$ OVTDM system model 


\section{SeVERAl Comparisons OF Multiple LDPC Code With OFDM AND OVXDM SYSTEMS}

The project team tried to build a multi-domain LDPC code with these technologies, which respectively built the corresponding system model. Due to limited space, three typical representatives are selected.

\section{A. Multiple LDPC Code Combined with OFDM}

The system model is shown in Figure 3.

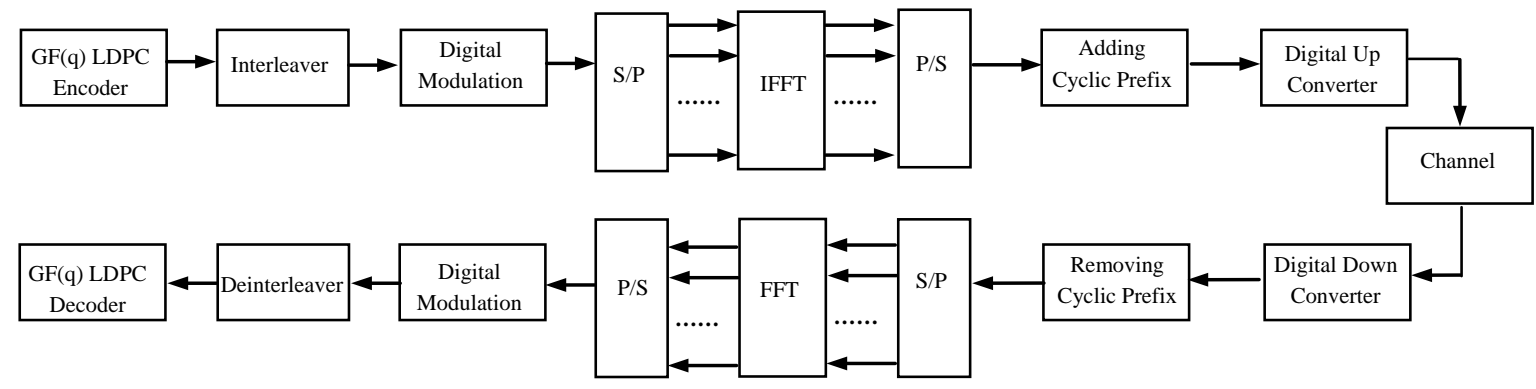

Figure 3. $\quad$ System model of multiple LDPC code combined with OFDM

At the sending end, the digital signal sent by the source first passes through the multiplex LDPC encoder. In order to prevent the fading channel from causing sudden errors, the coded code word needs to be interleaved and then input into the channel. The interleaved code character is made into the level symbol transmitted in the channel through digital modulation. Then S/P conversion and IFFT are performed to modulate the data symbols onto the corresponding subcarriers. After P/S conversion and adding cycle prefix, digital up conversion, and then it is sent into the channel. On the receiving end, the reverse works similarly[4].

\section{B. Multiple LDPC Code Combined with OVCDM}

The system model is shown in Figure 4. In this case, the OFDM part in Figure 3 is replaced with OVCDM module, and the coding part is consistent with Figure 1, but the decoding part adopts MAP algorithm[5].

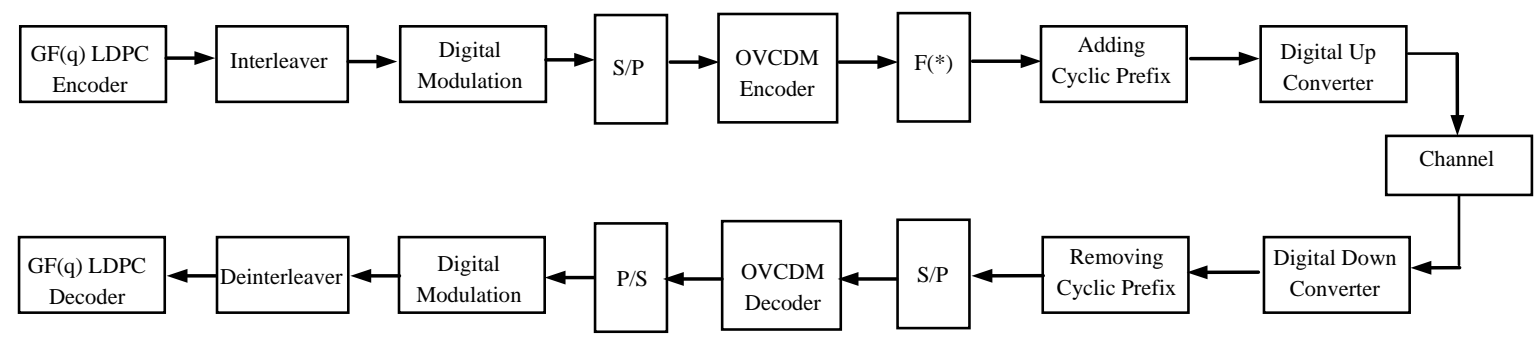

Figure 4. System model of multiple LDPC code combined with OVCDM

In OVCDM, the overlap between code words is considered as the mutual constraint between code words, so the only interference source in the whole system is external interference factor. For example, in AWGN channel, the interference of the system is white Gaussian noise.

The overlapping of symbols can make data symbols consistent, but it break the one-to-one correspondence between symbols. When considering data sequences, there is no such limitation. Channel coding can be regarded as the mapping between source sequence and coding sequence. Therefore, the decoding of OVCDM can be considered as a maximum likelihood sequence detection (MLSD).In the sense of minimal sequence error, MLSD is an optimal detection. When the prior probability information about the transmitted symbol exists, MLSD evolves into a detection algorithm under the MAP criterion. 


\section{Multiple LDPC Code Combined with OVTDM}

The system model is shown in Figure 5. In this case, the OFDM part in Figure 3 is replaced with OVTDM module, and the coding part is consistent with Figure 2, but the decoding part adopts maximum likelihood sequence detection (MLSD)[6].

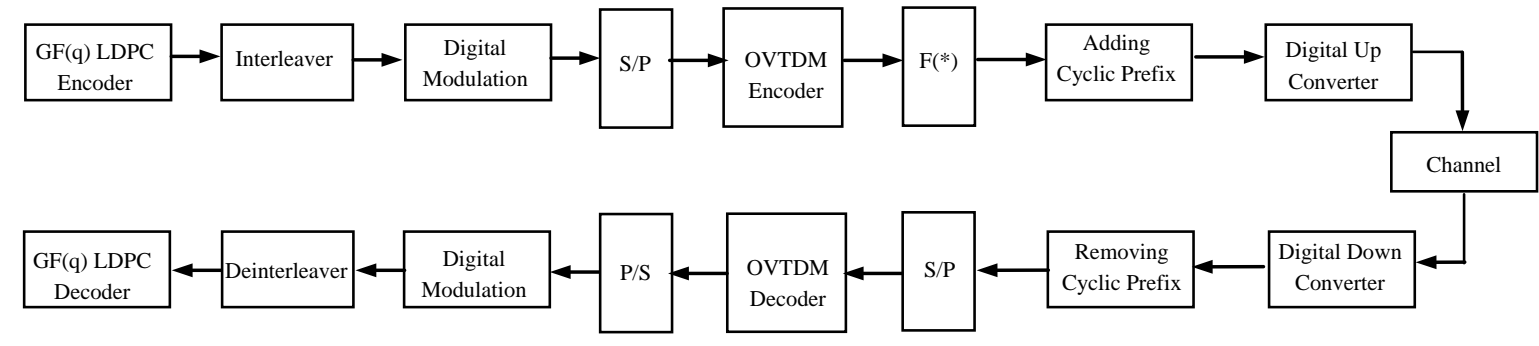

Figure 5. S S System model of multiple LDPC code combined with OVTDM

\section{Conclusion}

Based on the excellent performance of multi-domain LDPC code and combined with the characteristics of OFDM, OVCDM and OVTDM, the project team tries to build a system model that combines multi-domain LDPC code with these technologies respectively, and tries to conduct indepth research on data transmission and coding, which will provide reference ideas for further enriching the theoretical framework of data coding and decoding.

\section{Acknowledgment}

This work was supported by the Special Funding Project of China Postdoctoral Science Foundation (Grants No. 2014T70967); Natural Science Research Key Project of Anhui Province Higher School (Grants No. KJ2017A630); Key Construction Discipline Project at College Level of Anhui Xinhua University (Grants No. zdxk201702); Institute Project at College Level of Anhui Xinhua University (Grants No. yjs201706); the Ninth Batch of Young and Middle-aged "Academic Leaders" Training Objects Project of Anhui Xinhua University (Grants No. 2018xxk14).

\section{References}

[1] HE Guang-hua, BAI Bao-ming, MA Xiao, etal. Low-complexity likelihood Probability Derivation Algorithm for Non-Binary LDPC-coded Modulation System[J]. Journal of Communication, 2013, 34(9): 84-91.

[2] Li Dao-ben. Waveform Coding Theory with High Spectral Efficiency--OVTDM and its Application[M]. Beijing: Science Press, 2013.

[3] Chen Xiao-nan. OVTDM Communication System Implementation and Related Issues[D]. Beijing: Beijing university of posts and telecommunications, 2019.

[4] Huang Ping. Coded Modulation Technique[M]. Beijing: Peking University Press, 2012.

[5] Gong Xue, Cai Xi, Chen Qing-chun. On the Performance of the OVCDM Technologies[C]. 2008 Western China Youth Communication Academic Conference Proceedings. Cheng Du: Sichuan communication society, 2008.

[6] Zhang Jing. Research On Recursive Overlapped Time Division Multiplexing and its Combination With Cooperative Communication[D]. Xi'an: Xi'an university of Science And Technology, 2014. 